\title{
Kissing lesion-from tongue to the palate
}

\begin{abstract}
Median rhomboid glossitis presents as a well-defined erythematous area seen along the midline towards the posterior aspect of dorsal surface of the tongue. It may have a "kissing lesion", in the area of the hard palate as a result of constant contact. Here is a case of 62 year old male patient having median rhomboid glossitis and kissing lesions on the hard palate.
\end{abstract}

Keywords: oral candidiasis, median rhomboid glossitis, kissing lesion, OPD, Candida hyphae
Volume 6 Issue I - 2018

\begin{abstract}
Reshma Suvarna, Prasanna Kumar Rao, Raghavendra Kini, Gowri P Bhandarkar, Roopashri Rajesh Kashyap,Vidya holla Department of Oral Medicine and Radiology, AJ Institute of Dental Sciences, India
\end{abstract}

\begin{abstract}
Correspondence: Reshma Suvarna, Postgraduate student, Department of Oral Medicine and Radiology,AJ Institute of Dental Sciences, Kuntikana, NH-66, Mangaluru, PIN- 575004, Karnataka, India, Tel +919449966216,

Email itsreshma_II@yahoo.co.in
\end{abstract}

Received: December 01, 2017 | Published: March 02, 2018
Abbreviations: MRG, median rhomboid glossitis; CPA, central papillary atrophy of the tongue; LAT, localized atrophy of the tongue papillae; ATP, atrophy of the tongue papillae

\section{Case report}

A medically fit 62-year-old male patient walked into the OPD for a routine dental check up. Patient gave history of burning sensation over the posterior region of palate while eating spicy food since seven years. History of chewing paan masala with tobacco since three years 3-4 times per day. There was no relevant family history and no known history of allergies and medication. Patient was moderately built and nourished and his vital signs were within normal limit. Intraoral examination revealed well demarcated, red, depapillated, rhomboid lesion approximately $3 \times 2 \mathrm{cms}$ in size present on the dorsal surface of the tongue on midline just one $\mathrm{cm}$ anterior to the circumvallate

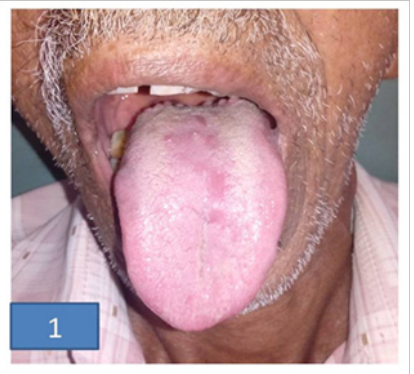

Figure I Red, depapillated lesion in the dorsal surface of the tongue (Median Rhomboid Glossitis).

\section{Discussion}

Median rhomboid glossitis (MRG) is defined as the central papillary atrophy of the tongue affecting $0.01 \%-1.0 \%$ of the total population. ${ }^{1}$ It was earlier thought to be a congenital abnormality related to the persistence of an embryonic midline tongue structure, the tubercular impar. However, the lesion is now believed to be a papillae (). On palpation the lesion was non tender, non-fluctuant and firm in consistency. Similar erythematous lesion approximately $3 \times 2$ $\mathrm{cm}$ in size with ill defined margins was present over the posterior part of the hard palate just opposite to the lesion present on the dorsal surface of the tongue. Lesion was non tender on palpation, soft in consistency and bleeding was absent (Figure 2). Based on history and clinical examination, a diagnosis of median rhomboid glossitis was made, and as this entity was concomitant with the palatal inflammation so a diagnosis of kissing lesions was given for the palatal lesions. Cytology and PAS staining confirmed the presence of Candida hyphae. Laboratory exams did not reveal anemia, diabetes, or HIV infection. Treatment with oral miconazole $20 \mathrm{mg}$ gel, TID, during four weeks, resolved significantly both lesions. The patient was advised to maintain proper oral hygiene.

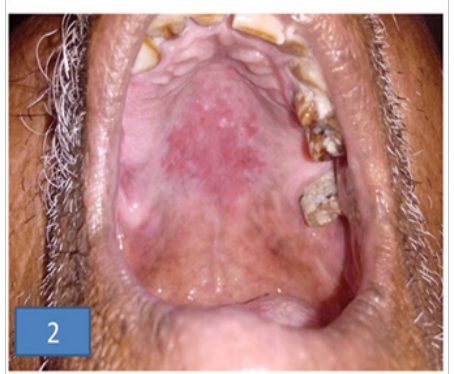

Figure 2 Kissing lesion on the posterior part of hard palate.

localized chronic infection by Candida albicans. Glossite lasangigue mediane de la face dorsal langue was the name given initially by Brocq and Pautrier. ${ }^{2}$ Other names given to the lesion are Central papillary atrophy of the tongue (CPA), localized atrophy of the tongue papillae (LAT), and atrophy of the tongue papillae (ATP). MRG is typically located around the midline of the dorsum of the tongue 
and is characterized by a shiny oval or diamond-shaped depapillated area on the midline of dorsal aspect of the tongue little anterior to the circumvallate papillae. ${ }^{2}$ Many of the cases are asymptomatic, some complain of persistent pain, irritation, or pruritus. ${ }^{3}$ When MRG is associated with palatal inflammation, it is called the kissing lesion; immune suppression should be suspected and investigated in these patients. This is considered as a marker of AIDS ${ }^{4}$ Despite frequent encounter of MRG, little is known about its etiology. Several predisposing factors such as smoking, denture wearing, diabetes mellitus, as well as candidal infections are associated with MRG. ${ }^{1,5}$ In a study conducted by Arendorf and Walker, it was reported that $44 \%$ of the total population harbour candidal organisms as part of their normal oral flora, and that tongue is the primary oral reservoir for Candida species . And mainly, the midline of the tongue is best for intense growth of Candida organisms. ${ }^{1}$

Another study by Whitaker and Singh said that as the tongue maintains close contact with the palatal mucosa during swallowing as well as at rest, the area of the tongue permanently contacts the palate. ${ }^{1,6}$ Also Farman discussed that an impaired blood supply to the mid-dorsal surface of the tongue might predispose it to the development of candidiasis and, more likely, to the consequent loss of filiform papillae. ${ }^{1}$ When MRG is seen along with palatal inflammation due to contact with the involved area on the tongue, it is known as kissing lesion; immune suppression should be suspected and it has been considered as a marker for AIDS. ${ }^{1,4}$ And sometimes nondissemination of infection is mostly due to the resistance of the palatal mucosa to adhesion of hyphae, and the lubricant and cleaning action of saliva. ${ }^{7}$ If the lesion shows ulceration or if it is solid on palpation, the possibility of malignant transformation should be considered and biopsy should be performed. ${ }^{1}$ Diagnosis of MRG is primarily clinical, although at times histopathology is required for differential diagnosis. Role of Candida can be proved by isolation of pathogenic Candida species from the lesion by laboratory techniques such as smear, culture of Candia on sabouraud's dextrose agar, colony forming units etc. The treatment is usually antifungal drugs followed by periodic clinical reassessments of the patient, as this type. Full remission after antimycotic therapy confirms the candidal infection. ${ }^{7}$

\section{Conclusion}

Median rhomboid glossitis was earlier considered to be a developmental defect. Today, however, it is believed to be a form of oral candidiasis. Questions concerning its importance and etiology still lingers. MRG in association with kissing lesion, immune suppression should be suspected and has also been considered as a marker for AIDS. Hence, proper diagnosis and management of these lesions are crucial.

\section{Acknowledgements}

None.

\section{Conflict of interest}

Authors declare there is no Conflict of interest towards this manuscript.

\section{References}

1. Goregen M, Miloglu O, Caglayan F, et al. Median Rhomboid Glossitis: A Clinical and Microbiological Study. European Journal of Dentistry. 2011;5(4):367-72.

2. Bihari M, Srivastava R, Jyoti B, et al. Median rhomboid glossitis with palatal 'kissing lesion'-A case report. Bangladesh. Journal of Dental Research \& Education. 2014;4(2):94-97.

3. Carter LC. Median rhomboid glossitis: Review of a puzzling entity. Compendium. 1990;11(7):448-451.

4. Rogers RS 3rd, Bruce AJ. The tongue in clinical diagnosis. J Eur Acad Dermatol Venereol. 2004;18(3):254-259.

5. Van der Wal N, Van der Kwast WA, van der Waal I. Median rhomboid glossitis: A follow-up study of 16 patients. J Oral Med. 1986;41(2):117-120.

6. Whitaker SB, Singh BB. Cause of median rhomboid glossitis. Oral Surg Oral Med Oral Pathol Oral Radiol Endod. 1996;81(4):379-380.

7. Galletta VC, Campos MS, Hirota SK, et al. Hyperplastic candidosis on the palate developed as a "kissing" lesion from median rhomboid glossitis. Braz J Otorhinolaryngol. 2010;76(1):137. 